\title{
Claudin-5-Binders Enhance Permeation of Solutes across the Blood-Brain Barrier in a Mammalian Mode[ ${ }^{\mathbb{\Phi}}$
}

\author{
Yosuke Hashimoto, Keisuke Shirakura, Yoshiaki Okada, Hiroyuki Takeda, Kohki Endo, \\ Maki Tamura, Akihiro Watari, Yoshifusa Sadamura, Tatsuya Sawasaki, Takefumi Doi, \\ Kiyohito Yagi, and Masuo Kondoh \\ Graduate School of Pharmaceutical Sciences, Osaka University, Osaka, Japan (Y.H., K.S., Y.O., A.W., T.D., K.Y., M.K.); \\ Proteo-Science Center, Ehime University, Ehime, Japan (H.T., T.S.); and Life Science Research Laboratories, \\ Wako Pure Chemical Industries, Ltd., Hyogo, Japan (K.E., M.T., Y.S.)
}

Received May 20, 2017; accepted August 7, 2017

\section{ABSTRACT}

A current bottleneck in the development of central nervous system (CNS) drugs is the lack of drug delivery systems targeting the CNS. The intercellular space between endothelial cells of the blood-brain barrier (BBB) is sealed by complex protein-based structures called tight junctions (TJs). Claudin-5 (CLDN-5), a tetra-transmembrane protein is a key component of the TJ seal that prevents the paracellular diffusion of drugs into the CNS. In the present study, to investigate whether
CLDN-5 binders can be used for delivery of drugs to the CNS, we generated monoclonal antibodies (mAbs) specific to the extracellular domains of CLDN-5. In an in vitro model of the BBB, the anti-CLDN-5 mAbs attenuated trans-epithelial/ endothelial electrical resistance and enhanced solute permeation. These anti-CLDN-5 mAbs are potential leads for the development of novel drug delivery systems targeting the CNS.

\section{Introduction}

The regulatory success rate for central nervous system (CNS) drugs is less than half that of non-CNS drugs (Kaitlin, 2014). Current bottlenecks in the development of CNS drugs include a delay in the development of conventional experimental models to accurately predict the efficacy of drug candidates in vivo and a lack of drug delivery systems to efficiently deliver drugs to the CNS (Pardridge, 2005; Gribkoff and Kaczmarek, 2017). Recent progress in genome editing and induced pluripotent stem cell technologies has led to the development of several experimental models useful for the development of CNS drugs (Heidenreich and Zhang, 2016; Liu and Deng, 2016; Gribkoff and Kaczmarek, 2017); however, the efficient delivery of drugs to the CNS remains a challenge.

This work was supported by a Health and Labour Sciences Research Grant from the Ministry of Health, Labour and Welfare of Japan; a research grant from the Japan Agency for Medical Research and Development; a Grant-in-Aid for Scientific Research from the Ministry of Education, Culture, Sports, Science and Technology of Japan [Grant number 24390042]; and funds from the Adaptable and Seamless Technology Transfer Program through Target-driven R\&D Agency; Platform for Drug Discovery, Informatics, and Structural Life Science of the Ministry of Education, Culture, Sports, Science and Technology of Japan; and the Takeda Science Foundation. Y.H. was supported by a Research Fellowship for Young Scientists from the Japan Society for the Promotion of Science [Grant number 15J10065]

https://doi.org/10.1124/jpet.117.243014.

S This article has supplemental material available at jpet.aspetjournals.org.
The blood-brain barrier (BBB) is a membrane barrier formed by brain capillary endothelial cells that protects the brain from harmful materials circulating in the blood (Rubin and Staddon, 1999). Ninety-eight percent of small-molecule drugs and nearly all large-molecule drugs, including antibodies, recombinant proteins, and antisense therapeutic agents, do not readily cross the BBB (Pardridge, 2005). Drugs that readily cross the $\mathrm{BBB}$ do so via either transcellular transport (i.e., through cell bodies via receptors and transporters in the cell membrane) or paracellular transport (i.e., through the intercellular space between cells) (Laksitorini et al., 2014). To date, most studies aimed at enhancing the delivery of drugs across the BBB have focused on transcellular transport-based methods such as increasing the passive diffusion of drugs by altering their physicochemical properties (Betz et al., 1980; Lipinski et al., 2001) or by using receptormediated transport and carrier proteins such as transferrin receptors (Pardridge, 2007; Pardridge and Boado, 2012).

Tight junctions (TJs) are complex biochemical structures that seal the intercellular space between adjacent endothelial cells, which prevents the diffusion of solutes from the systemic circulation into the CNS via the BBB. Thus, the strategy underlying the delivery of drugs to the brain via paracellular transport is to disrupt, or open, the TJ seal in the BBB (Pardridge, 2005). Osmotic opening of the TJ seal by the injection of mannitol has been used clinically to deliver

ABBREVIATIONS: BBB, blood-brain barrier; C-CPEmt, C-terminal half of Clostridium perfringens enterotoxin mutant; CLDN, claudin; CNS, central nervous system; DMEM, Dulbecco's modified Eagle's medium; ECL, extracellular loop domain; FBS, fetal bovine serum; FD-4, fluorescein isothiocyanate-labeled dextran with an average weight of $4 \mathrm{kDa}$; $\mathrm{mAb}$, monoclonal antibody; $\mathrm{P}_{\mathrm{app}}$, apparent permeability; PBS, phosphate-buffered saline; TBST, Tris-buffered saline containing 0.05\% Tween-20; TEER, trans-epithelial/endothelial electrical resistance; TJ, tight junction; ZO, zonula occludens. 
anticancer drugs directly to brain tumors, with transient adverse effects, such as the leakage of albumin into the brain, but without clinically significant adverse effects (Rapoport, 2000). In addition, phase $1 / 2 \mathrm{a}$ studies have revealed that repeated opening of the BBB by using a pulsed ultrasound system is safe and well tolerated in patients with brain tumors (Carpentier et al., 2016). However, the potential applications of this method are limited because it opens the BBB to all large molecules, not just the target drug (Laksitorini et al., 2014).

TJs are composed of transmembrane proteins (e.g.,junctional adhesion molecules, occludin, claudins [CLDNs], tricellulin, angulins) and intracellular adaptor proteins (e.g., zonula occludens [ZO]) (Furuse, 2014; Zihni et al., 2016). CLDNs, which comprise a 27-member protein family of tetratransmembrane proteins, are the major structural and functional components of the TJ seal (Markov et al., 2015). The expression profile and $\mathrm{TJ}$-sealing functions of the CLDNs differ among tissues. However, it has been reported that the BBB is more permeable to molecules with a molecular weight of $<800 \mathrm{Da}$ in CLDN-5-deficient mice than in wild-type mice (Nitta et al., 2003). In addition, knockdown of Cldn-5 in mice leads to accumulation of small-molecular-weight molecules (562 Da) in the brain (Campbell et al., 2008). Together, these findings suggest that CLDN-5 is a potential target for targeted delivery of drugs to the CNS via paracellular transport.

Here, we generated four anti-CLDN-5 monoclonal antibodies (mAbs) and examined the effects of these mAbs on the integrity of the TJ seal and the permeation of solutes in a model of the mammalian BBB.

\section{Materials and Methods}

Animals. Male BXSB mice (6 weeks old), female BALB/c nu/nu mice ( 6 weeks old), and female Wistar rats (6 weeks old) were purchased from Shimizu Laboratory Supplies (Kyoto, Japan). All animals were maintained under controlled conditions of a 12-hour light/dark cycle at $23 \pm 1.5^{\circ} \mathrm{C}$. The rodents were given ad libitum access to food and water. Animal experiments were approved by Osaka University (Osaka, Japan).

Cells. L cells stably expressing mouse CLDN-5 were provided by Dr. S. Tsukita (Kyoto University, Kyoto, Japan). HT-1080 cells stably expressing human CLDN-1, CLDN-2, CLDN-3, CLDN-4, CLDN-5, CLDN-6, or CLDN-7 were developed as described previously (Hashimoto et al., 2016). P3U1 mouse myeloma cells, MDCKII cells, Phoenix-A packaging cells, and Caco-2 and T84 human intestinal cells were purchased from American Type Culture Collection (Manassas, VA).

HT-1080 cells (a cell line of human fibrosarcoma) are used for the characterization of CLDN binders because HT-1080 cells are CLDNnegative cells (Neesse et al., 2013; Fukasawa et al., 2015; Mosley et al., 2015; Nakajima et al., 2015). MDCKII cells (a cell line of canine epithelial cell) are a representative model for the characterization of CLDN-5-based paracellular permeation and tight junction integrity (Wen et al., 2004; Amasheh et al., 2005; Piehl et al., 2010; Del Vecchio et al., 2012). HT-1080 cells and MDCKII cells expressing various CLDNs were prepared as described previously with minor modifications (Hashimoto et al., 2016). cDNAs encoding cynomolgus monkey CLDN-5 and two human CLDN-5/human CLDN-1 chimeric mutants were commercially synthesized by Invitrogen (Carlsbad, CA) and inserted into pCX4pur vector (Akagi et al., 2000). The cDNAs encoding three human CLDN-5/mouse CLDN-5 chimeric mutants were generated by means of site-directed mutagenesis of pCX4pur encoding human CLDN-5. The resulting vectors were transfected into Phoenix-A cells by using the X-tremeGENE HP DNA Transfection Reagent (Roche Diagnostics, Mannheim, Germany), and the retrovirus-containing supernatant was harvested 48 hours after transfection. The retrovirus-containing supernatant was mixed with $8 \mu \mathrm{g} / \mathrm{ml}$ Polybrene (hexadimethrine bromide; Sigma-Aldrich, Melbourne, Victoria, Australia) and used to transduce HT-1080 or MDCKII cells. Stably transduced cells were selected by using $5 \mu \mathrm{g} / \mathrm{ml}$ puromycin (InvivoGen, San Diego, CA).

L cells, HT-1080 cells, MDCKII cells, and Phoenix-A cells were maintained in Dulbecco's modified Eagle's medium (DMEM) supplemented with $10 \%$ heat-inactivated fetal bovine serum (FBS) (Nichirei Biosciences, Tokyo, Japan), $100 \mathrm{U} / \mathrm{ml}$ penicillin, and $100 \mu \mathrm{g} / \mathrm{ml}$ streptomycin (Nacalai Tesque, Kyoto, Japan). P3U1 cells were maintained in RPMI 1640 medium supplemented with 10\% heat-inactivated FBS, $100 \mathrm{U} / \mathrm{ml}$ penicillin, and $100 \mu \mathrm{g} / \mathrm{ml}$ streptomycin. All hybridomas were maintained in Hybridoma-SFM Medium (Gibco, Grand Island, NY) supplemented with 10\% BM Condimed H1 (Roche Diagnostics). Caco-2 cells were maintained in DMEM supplemented with $10 \%$ FBS, $100 \mathrm{U} / \mathrm{ml}$ penicillin, and $100 \mu \mathrm{g} / \mathrm{ml}$ streptomycin. T84 cells were maintained in a 1:1 mixture of DMEM and Ham's F-12 medium supplemented with $10 \% \mathrm{FBS}, 100 \mathrm{U} / \mathrm{ml}$ penicillin, and $100 \mu \mathrm{g} / \mathrm{ml}$ streptomycin. All cells were incubated at $37^{\circ} \mathrm{C}$ under $5 \% \mathrm{CO}_{2}$.

Generation and Purification of Anti-CLDN-5 mAbs. Male BXSB mice and female Wistar rats were immunized every 2 weeks for 8 weeks with a eukaryotic expression vector encoding human CLDN-5, which is proprietary technology of GENOVAC GmbH (Freiburg, Germany). To generate hybridoma cells, lymphocytes were harvested 7 days after the final immunization and fused with P3U1 cells by using polyethylene glycol 1000 (Roche Diagnostics). Hybridoma cells producing antibodies that reacted with human CLDN-5 were screened for the selectivity of their antibodies to bind to HT-1080/human CLDN-5 but not HT-1080/mock cells. The mAb subclass was determined by using a mouse or rat Ig-isotyping enzyme-linked immunosorbent assay kit (BD Biosciences, Franklin Lakes, NJ).

Purified mAbs were prepared as described previously (Hashimoto et al., 2016). Briefly, hybridoma cells were inoculated intraperitoneally into pristane-injected female BALB/c nu/nu mice, resulting in the production of ascitic fluid containing the mAbs. The mAbs were purified from the ascitic fluid by using Protein G Sepharose 4 Fast Flow Columns (GE Healthcare, Princeton, NJ). The purified mAbs were then dialyzed against phosphate-buffered saline (PBS; $\mathrm{pH} 7.4$ ) and stored at $-30^{\circ} \mathrm{C}$. The concentration of mAbs was quantified with a BCA protein assay kit using bovine serum albumin as the standard (Pierce Chemical, Rockford, IL).

Preparation of C-terminal half of a Clostridium perfringens Enterotoxin Mutant. A mutant of the C-terminal half of a Clostridium perfringens enterotoxin [C-CPEmt $\left(\mathrm{C}-\mathrm{CPE}_{\mathrm{Y} 306 \mathrm{~W} / \mathrm{S} 313 \mathrm{H}}\right)$ ] that recognizes a broad range of CLDNs, including CLDN-5, was prepared as described previously with minor modifications (Takahashi et al., 2012; Protze et al., 2015). Briefly, Escherichia coli BL-21 (DE 3) was transformed with the pET16b vector encoding C-CPEmt, and protein expression was induced by the addition of isopropyl-D-thiogalactopyranoside. The cells were then harvested and lysed in buffer A [10 mM Tris-HCl (pH 8.0), $400 \mathrm{mM} \mathrm{NaCl}, 5 \mathrm{mM} \mathrm{MgCl}_{2}, 0.1 \mathrm{mM}$ phenylmethanesulfonyl fluoride, $1 \mathrm{mM} 2$-mercaptoethanol, and $10 \%$ glycerol]. The $10 \times$ Histagged C-CPEmt was isolated from the cell lysate by means of affinity chromatography with an immobilized metal affinity chromatography column (HisTrap HP column; GE Healthcare). The buffer was exchanged with PBS by gel filtration (PD-10; GE Healthcare) and stored at $-80^{\circ} \mathrm{C}$. The concentration of C-CPEmt was quantified with a BCA protein assay kit (Pierce Chemical).

Flow Cytometric Analysis. To analyze the binding specificity of the anti-CLDN-5 mAbs, cells expressing the various CLDNs were detached from the culture plates. The cells were then incubated with the supernatants of hybridomas or with purified mAbs $(5 \mu \mathrm{g} / \mathrm{ml})$ and then were stained with fluorescence-conjugated goat anti-mouse or anti-rat IgG (Jackson ImmunoResearch, West Grove, PA). A mixture of C-CPEmt and anti-His tag antibody (Sigma-Aldrich) was used as the positive control.

Western Blotting. To examine the expression level of several TJ proteins in CLDN-5-expressing HT-1080 and MDCKII cells, Caco-2 
cells, and T84 cells, cell lysates were subjected to SDS-PAGE and then transferred to polyvinylidene difluoride membranes. After blocking with 5\% skimmed milk containing TBST (Tris-buffered saline containing $0.05 \%$ Tween-20), the membranes were incubated with rabbit anti-ZO-1 (catalog \#40-2200; Invitrogen), rabbit anti-occludin (catalog \#71-1500; Invitrogen), rabbit anti-CLDN-1 (catalog \#51-9000; Invitrogen), rabbit anti-CLDN-2 (catalog \#51-6100; Invitrogen), rabbit anti-CLDN-3 (catalog \#34-1700; Invitrogen), mouse anti-CLDN-4 (catalog \#32-9400; Invitrogen), or rabbit anti-CLDN-5 (catalog \#SAB4502981; Sigma-Aldrich) in $2 \%$ skimmed milk containing TBST for 12 hours at $4^{\circ} \mathrm{C}$. Mouse anti- $\beta$-actin (AC-15; Sigma-Aldrich) was used as the loading control.

After incubation of the primary antibodies, the membranes were incubated with a horseradish peroxidase-conjugated anti-mouse or anti-rabbit antibody (Jackson ImmunoResearch) in TBST and then incubated for 2 hours at room temperature. After washing with TBST, the membranes were treated with Chemi-Lumi Super (Nacalai Tesque) to detect the horseradish peroxidase, and chemiluminescent signals were imaged with an LAS4100 imager (GE Healthcare).

Measurement of the Electrical Resistance of Cell Monolayers. Trans-epithelial/endothelial electrical resistance (TEER), which reflects the integrity of the TJ seal, was measured by using a Millicell ERS Ohmmeter (Millipore, Eschborn, Germany) and a culture plate warmer.

To measure the TEER of monolayers of CLDN-5-expressing MDCKII cells, $0.8 \times 10^{5}$ mock MDCKII cells or MDCKII cells expressing human, cynomolgus monkey, or mouse CLDN-5 were seeded onto Falcon Cell Culture Inserts (polyester membrane, $0.4 \mu \mathrm{m}$ pore size, $0.3 \mathrm{~cm}^{2}$ culture area; BD Biosciences) and cultured for 1 week (top compartment, $0.3 \mathrm{ml}$; lower compartment, $0.9 \mathrm{ml}$ ). After the exchange of $90 \mu \mathrm{l}$ of medium from the bottom compartment with $90 \mu \mathrm{l}$ of a $900 \mu \mathrm{g} / \mathrm{ml} \mathrm{mAb}$ in PBS (final concentration, $90 \mu \mathrm{g} / \mathrm{ml}$ ), the TEER of the monolayer was measured for 12 hours. Treatments with PBS (vehicle), mouse IgG, or rat IgG were used as negative controls, and treatment of the bottom compartment with $60 \mu \mathrm{g} / \mathrm{ml} \mathrm{C-CPEmt}$ was used as the positive control.

To measure the TEER of a monolayer of cynomolgus monkey brain microvasculature cells, a triple coculture model (PharmaCo-cell, Nagasaki, Japan) was cultured for 5 days (Nakagawa et al., 2009). After the exchange of $30 \mu \mathrm{l}$ of medium from the top compartment with $30 \mu \mathrm{l}$ of a $1500 \mu \mathrm{g} / \mathrm{ml} \mathrm{mAb}$ in PBS (final concentration, $150 \mu \mathrm{g} / \mathrm{ml}$ ), the TEER of the monolayer was measured for 12 hours. Treatment of the top compartment with $100 \mu \mathrm{g} / \mathrm{ml} \mathrm{C-CPEmt} \mathrm{was} \mathrm{used} \mathrm{as} \mathrm{the} \mathrm{positive}$ control.

To measure the TEER of a monolayer of Caco-2 or T84 human intestinal cells, cells $\left(0.8 \times 10^{5}\right.$ cells $)$ were seeded into Falcon Cell Culture Inserts (BD Biosciences) and cultured for 10 days. After exchange of 30 and $90 \mu \mathrm{l}$ of medium from the top and bottom compartments with 30 and $90 \mu \mathrm{l}$ of a $1500 \mu \mathrm{g} / \mathrm{ml} \mathrm{mAb}$ in PBS, respectively (final concentration, $150 \mu \mathrm{g} / \mathrm{ml}$ ), the TEER of the monolayers was measured for 12 hours. Treatments with PBS (vehicle), mouse IgG, and rat IgG were used as negative controls, and treatment with $100 \mu \mathrm{g} / \mathrm{ml} \mathrm{C-CPEmt} \mathrm{in} \mathrm{both} \mathrm{compartments} \mathrm{was}$ used as the positive control.

Measurement of the Permeation of Solutes through Cell Monolayers. After measurement of the TEER of monolayers of cynomolgus monkey brain microvasculature cells, the permeability of the monolayers to sodium fluorescein (376 Da; Wako Pure Chemical, Osaka, Japan) or fluorescein isothiocyanate-labeled dextran with an average molecular weight of $4 \mathrm{kDa}$ (FD-4; Sigma-Aldrich) was determined. After washes with DMEM (without FBS), cell culture inserts were transferred to 24-well plates (Grainer, Frickenhausen, Germany) containing $0.9 \mathrm{ml}$ of DMEM (without FBS). The medium in the top chamber was then changed to $0.2 \mathrm{ml}$ of $10 \mu \mathrm{g} / \mathrm{ml}$ sodium fluorescein or $1 \mathrm{mg} / \mathrm{ml} \mathrm{FD-4} \mathrm{in} \mathrm{DMEM} \mathrm{(without} \mathrm{FBS),} \mathrm{and} \mathrm{the} \mathrm{culture}$ inserts were incubated for 30 minutes on a plate warmer. Samples were collected from the bottom compartment, and the concentration of the tracer was measured using a TriStar LB 941 plate reader
(Berthold Technologies, Bad Wildbad, Germany). Apparent permeability $\left(\mathrm{P}_{\mathrm{app}}\right)$ coefficients (in centimeters per second) were calculated by using the following equation:

$$
\mathrm{P}_{\text {app }}=\frac{\text { volume of lower compartment } \times \text { concentration of tracer in the sample }}{\text { assay time } \times \text { surface area of the culture inserts } \times \text { initial tracer concentration }}
$$

Statistical Analysis. Data were analyzed by using one-way analysis of variance followed by Bonferroni post hoc test. Statistical significance for all comparisons was set at $P<0.05$.

\section{Results}

Generation and Characterization of mAbs Targeting the Extracellular Loop Domains of Human CLDN-5. Previously, we generated anti-CLDN-1 and anti-CLDN-4 mAbs by using DNA immunization methods (Fukasawa et al., 2015; Hashimoto et al., 2016). In the present study, we used these techniques to generate anti-CLDN-5 mAbs by immunizing BXSB mice and Wistar rats with a plasmid encoding human CLDN-5. Four mAbs targeting the extracellular loop domains (ECLs) of human CLDN-5 were isolated: M11 and M48 from the mice and R2 and R9 from the rats (Fig. 1A). All four mAbs specifically were bound to CLDN-5 but not to CLDN-1 to CLDN-4, CLDN-6, or CLDN-7 (Fig. 1B). A crossspecies reactivity analysis of the $\mathrm{mAbs}$ revealed that all four mAbs was bound to human and cynomolgus monkey CLDN-5 but not to mouse CLDN-5, except for R9, which also was weakly bound to mouse CLDN-5 (Fig. 1C).

We next investigated the epitopes of CLDN-5 recognized by the anti-CLDN-5 mAbs by swapping amino acids 26-78 in ECL1 and amino acids 147-162 in ECL2 of human CLDN-5 with the corresponding amino acids in human CLDN-1 (chimeric mutants $1-5$, and 5-1, respectively) (Fig. 2A). C-CPEmt, which is a broadly specific binder of CLDN-1 to CLDN-7, was bound to these mutants (Fig. 2B), indicating that these chimeric mutants had normal tropism as transmembrane proteins and were correctly recruited to the cell membrane. However, the substitution of ECL1 of human CLDN-5 with that of human CLDN-1 (chimeric mutant 1-5) attenuated the binding of M11 and R2, but not that of M48 or R9 (Fig. 2B), and the substitution of ECL2 of human CLDN-5 with that of human CLDN-1 (chimeric mutant 5-1) attenuated the binding of M48 and R9, but not that of M11 or R2 (Fig. 2B). These findings suggested that the epitope for M11 and R2 was contained in ECL1, whereas that for M48 and R9 was contained in ECL2 (Table 1). Alignment of the amino acid sequences of human, cynomolgus monkey, and mouse CLDN-5 revealed the following three candidate epitopes (Fig. 2C): aspartic acid (D) at position 68 in ECL1; threonine (T) at position at 75 in ECL1; and serine (S) at position 151 in ECL2. Therefore, we next generated transfectants stably expressing mutant human CLDN-5 containing the substitutions D68E, T75A, and S151T (Fig. 2D). The D68E substitution attenuated the binding of M11 and R2, and the S151T substitution partly attenuated the binding of M48 and R9 (Fig. 2E). These results suggest that in human CLDN-5, the dominant epitope of M11 and R2 is D68 in ECL1 and that of M48 and R9 is S151 in ECL2 (Table 1).

Effects of the Anti-CLDN-5 mAbs on TJ Integrity in MDCKII Transfectants. Next, to investigate whether the anti-CLDN-5 mAbs decreased TJ integrity in a CLDN-5dependent manner, MDCKII transfectants expressing human, cynomolgus monkey, or mouse CLDN-5 were established (Fig. 3A). TJ integrity, as determined by TEER, 

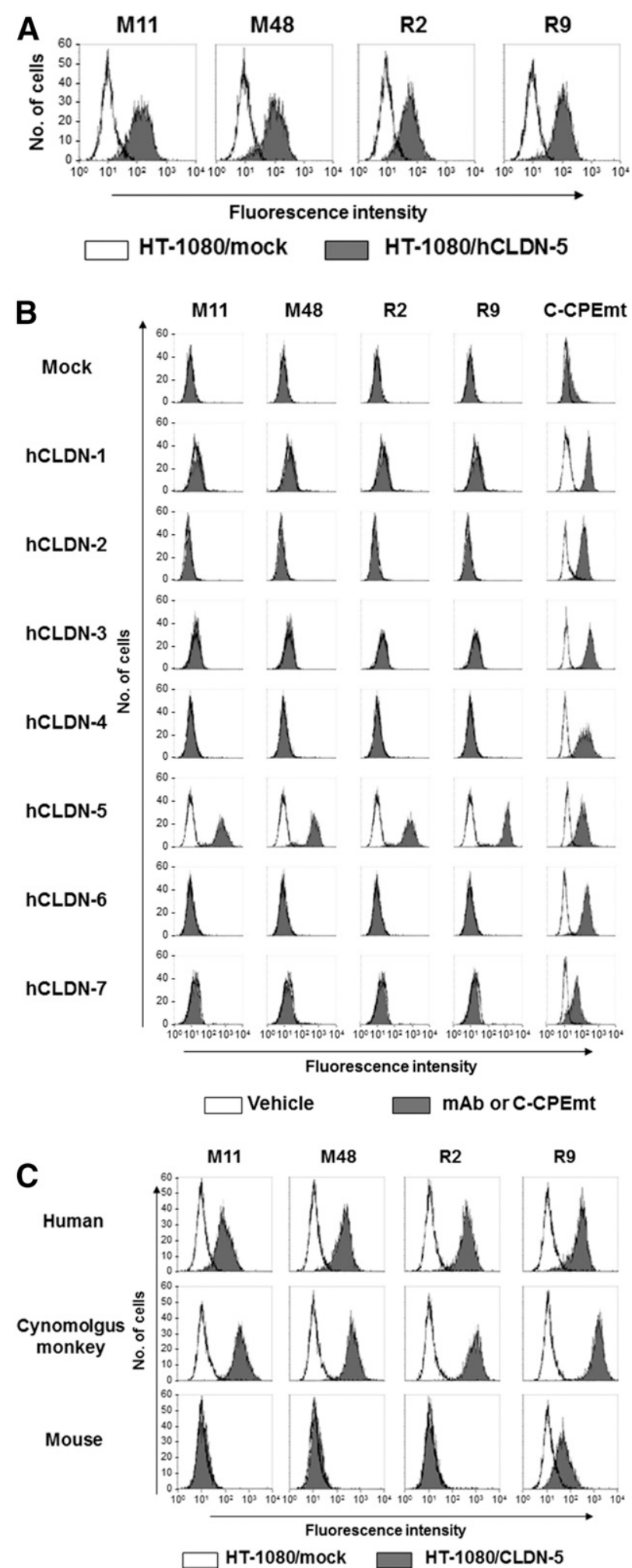

Fig. 1. Generation and characterization of mAbs targeting human CLDN-5. (A) Flow cytometric analysis. Mock-transfected or human CLDN-5-expressing HT-1080 cells were treated with the four generated mAbs (M11, M48, R2, or R9). The cells were then treated with fluorescein-labeled secondary antibodies, and mAb-bound cells were detected by flow cytometry. (B) CLDN specificity of the mAbs. Mock-transfected or human CLDNexpressing HT-1080 cells were treated with the indicated mAbs or the C-CPEmt. The cells were then treated with fluorescein-labeled secondary antibodies or anti-His-tag mAbs followed by fluorescein-labeled antibodies. mAb- or C-CPEmt-bound cells were detected by flow cytometry. C-CPEmt binding was used as the positive control. (C) Species specificity peaked at 20-30, 230-250, 250-270, and 400-420 $\Omega / \mathrm{cm}^{2}$ in MDCKII/mock, MDCKII/hCLDN-5, MDCKII/cCLDN-5, and MDCKII/mCLDN-5 cells, respectively (Supplemental Fig. 1). Treatment of all transfectants with C-CPEmt decreased TJ integrity (Fig. 3B). All four of the anti-CLDN-5 mAbs time-dependently decreased TEER in the hCLDN-5and cCLDN-5-expressing transfectants. In contrast, none of the anti-CLDN-5 mAbs affected TJ integrity in the mCLDN-5-expressing transfectant (Fig. 3B). These findings suggest that M11, M48, R2, and R9 decreased TJ integrity by interacting with CLDN-5. TEER values returned to baseline 24 hours after the anti-CLDN-5 mAbs were removed (Supplemental Fig. 2). The binding affinity of R9 to mouse CLDN-5 may not have been strong enough to decrease TJ integrity in the mouse CLDN-5 transfectant (Supplemental Fig. 3).

Next, we investigated the effects of the anti-CLDN-5 mAbs on the BBB by using a commercially available BBB model composed of a coculture of cynomolgus monkey brain microvasculature endothelial cells, rat pericytes, and rat astrocytes (Nakagawa et al., 2009). Treatment of the model BBB with the anti-CLDN-5 mAbs time-dependently reduced TEER (Fig. 4A). In addition, no cytotoxicity was observed after treatment with the anti-CLDN-5 mAbs (Supplemental Fig. 4). M48 and R9 modulated TJ integrity more than did M11 and R2, suggesting that binders targeting ECL2 of CLDN-5 may modulate TJ integrity more than binders targeting ECL1.

Next, we clarified whether the anti-CLDN-5 mAbs increased the permeability of the model BBB to solutes by using a fluorescein dye with a molecular mass of $376 \mathrm{Da}$ and FD-4. Control IgG did not increase the permeability of the model BBB to the fluorescein dye or FD-4 (Fig. 4, B and C). However, treatment with the anti-CLDN-5 mAbs significantly increased the permeability of the model $\mathrm{BBB}$ to the fluorescein dye and FD-4 compared with vehicle $(P<0.05)$ (Fig. 4 , B and C). R9 was the most potent permeation enhancer: $\mathrm{P}_{\mathrm{app}}$ of fluorescein dye: vehicle, $3.8 \times 10^{-6} \mathrm{~cm} / \mathrm{s}$ versus R9 $(150 \mu \mathrm{g} / \mathrm{ml}) 12.3 \times 10^{-6} \mathrm{~cm} / \mathrm{s}$; $\mathrm{P}_{\text {app }}$ of FD-4: vehicle, $1.0 \times 10^{-6} \mathrm{~cm} / \mathrm{s}$ versus R9 $(150 \mu \mathrm{g} / \mathrm{ml})$ $6.0 \times 10^{-6} \mathrm{~cm} / \mathrm{s}$ (Fig. $4, \mathrm{~B}$ and C).

To examine the cell specificity of the $\mathrm{TJ}$ integrity-modulating activity of the anti-CLDN-5 mAbs, we investigated the effect of the anti-CLDN-5 mAbs on TJ integrity in two human intestinal epithelium models: Caco-2 and T84 cells. Immunoblot analyses showed that both Caco-2 and T84 cells expressed CLDN-5, but the expression of CLDN-5 in the Caco-2 cells was much lower than that in the T84 cells (Fig. 5A). Although C-CPEmt decreased the TEER in monolayers of both Caco-2 and T84 cells, the anti-CLDN-5 mAbs did not, even at $150 \mu \mathrm{g} / \mathrm{ml}$ (Fig. 5B), suggesting that anti-CLDN-5 mAbs might be BBB-specific modulators.

\section{Discussion}

Although the concept of CLDN-5-targeted drug delivery to the brain was proposed more than a decade ago (Nitta et al., 2003), the concept remains unproven because no

of the mAbs. Human, cynomolgus monkey, or mouse CLDN-5-expressing HT-1080 cells were treated with the mAbs. The cells were then treated with fluorescein-labeled secondary antibodies, and $\mathrm{mAb}$-bound cells were detected by flow cytometry. 
CLDN-5-specific binders have been developed. Here, we used DNA immunization to generate CLDN-5-specific mAbs that targeted the extracellular domains of CLDN-5, and we found that these mAbs time- and dose-dependently decreased TJ integrity and increased the permeation of solutes across a model BBB.

CLDN-5 is ubiquitously expressed in the mammalian body, and CLDN-5-deficient mice die within 10 hours after birth
A
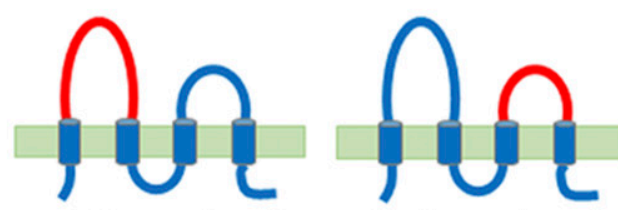

- hCLDN-5

1-5 mutant

\section{5-1 mutant}
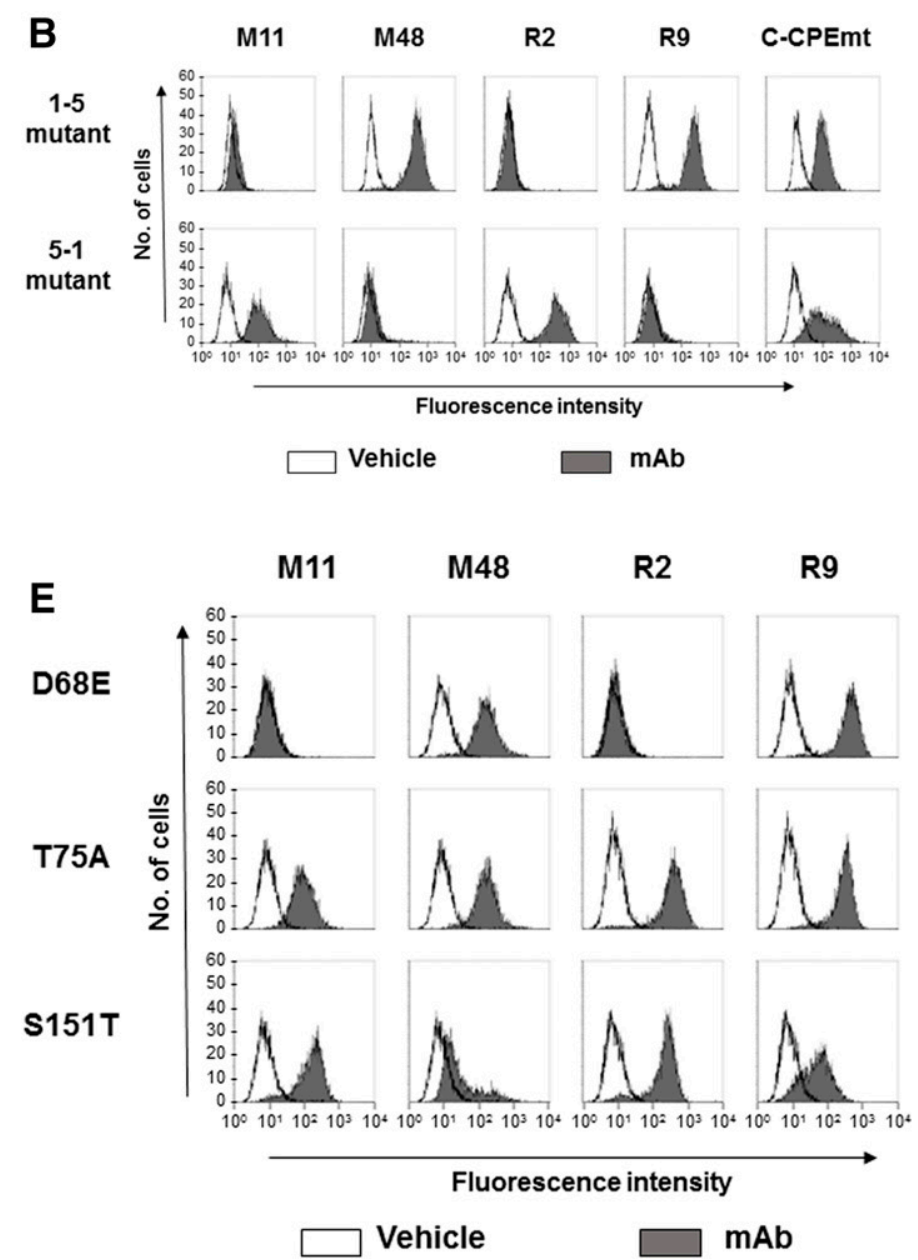

C

Human CLDN-5 MGSAALEILGLVLCLVGWGGLILACGLPMWOVTAFLDHNIVTAOTTWKGLWMSCVVOSTG Monkey CLDN-5 MGSAALEILGLVLCLVGWGL ILACGLPMWOVTAFLDHNI VTAOTTWKGLWMSCVVOSTG Mouse CLDN-5 MGSAALEILGLVLCLVGWVGLILACGLPMWOVTAFLDHNIVTAQTTWKGLWMSCVVOSTG

Human CLDN-5 HMOCKVID́SVLALSİEVOAARALTVSAVLLAFVALFVTLAGAOCTTCVAPGPAKARVALT Monkey CLDN-5 HMOCKVYSSVLALSTEVVAARALTVGAVLLAFVALFVTLAGAOCTTCVAPGPAKARVALT Mouse CLDN-5 HMOCKVYESVLALSAEVVQAARALTVGAVLLALVALFVTLTGAQCTTCVAPGPVKARVALT

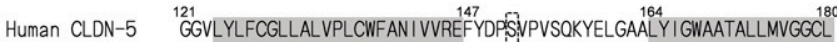
Monkey CLDN-5 GGVLYLLCGLLALVPLCWFANI VVREFYDPSSNPVSOKYELGAALYIGWAATALLMVGGG Mouse CLDN-5 GGALYAVCGLLALVPLCWFANI VVREFYDPITIVPVSOKYELGAALYIGWAASALLMCGGGL

Human CLDN-5 ${ }^{181} \stackrel{187}{18}$ LCGAVCTGRPLLSFPVKYSAPRRPTATGOYDKKNYY ${ }^{218}$ Monkey CLDN-5 LCCGAWVCTSRPDLSFPVKYSAPRRPTATGDYDKKNYV Mouse CLDN-5 VCCGAWVCTGRPEFSFPVKYSAPRRPTANGDYDKKNYV

D
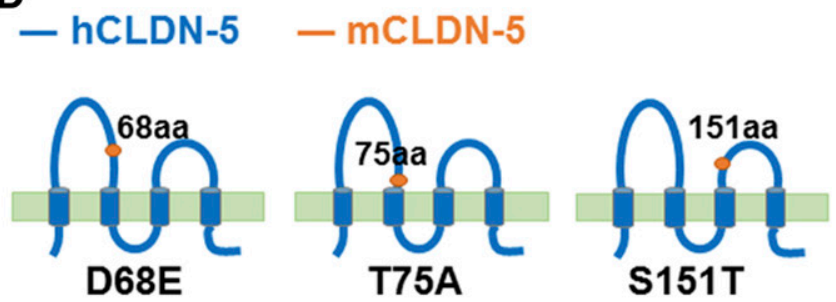

Fig. 2. Epitope mapping of the generated anti-CLDN-5 antibodies. (A) Schematic illustration of the human CLDN-5/CLDN-1 chimeric constructs. Green represents the cell membrane. Amino acids 26-78 and 147-162 of the ECLs of human CLDN-5 were swapped with the corresponding amino acids of human CLDN-1 to generate the 1-5 and 5-1 mutant, respectively. (B) Binding of the anti-CLDN-5 mAbs to human CLDN-5/CLDN-1 chimeric mutants. Chimeric mutant-expressing HT-1080 cells were treated with the anti-CLDN-5 mAbs or C-CPEmt, followed by incubation with fluoresceinlabeled antibodies or anti-His-tag mAbs and fluorescein-labeled antibodies, respectively. mAb- or C-CPEmt-bound cells were detected by flow cytometry. C-CPEmt was used as the positive control for the chimeric mutants. (C) Alignment of the amino acid sequences of human, cynomolgus monkey, and mouse CLDN-5. The sequences were obtained from the National Center for Biotechnology Information database (accession numbers: human, AAH19290.2; cynomolgus monkey, XP_005596069.1; and mouse, NP_038833.2). Gray highlights indicate putative transmembrane domains, predicted by the SOSUI program (http://harrier.nagahama-i-bio.ac.jp/sosui/). The amino acids at positions 68,75 , and 151 are enclosed within dashed lines. (D) Schematic illustration of the human/mouse CLDN-5 mutants. Human CLDN-5/mouse CLDN-5 chimeric CLDNs were generated by single amino acid substitution in the ECLs of human CLDN-5 with the corresponding amino acids in mouse CLDN-5 (mutants D68E, T75A, and S151T). (E) Binding of the anti-CLDN-5 mAbs to the human/mouse CLDN-5 chimeric mutants. Chimeric mutant-expressing HT-1080 cells were treated with the anti-CLDN-5 mAbs or C-CPEmt, followed by incubation with fluorescein-labeled antibodies or anti-His-tag mAbs and fluorescein-labeled mAbs, respectively. The mAb- or C-CPEmt-bound cells were detected by flow cytometry. C-CPEmt was used as the positive control for the chimeric mutants. 
TABLE 1

Summary of epitope mapping

\begin{tabular}{llcccccc}
\hline \multirow{2}{*}{ Clone } & \multirow{2}{*}{ Subclass } & \multicolumn{2}{c}{ Human CLDN-5/CLDN-1 Chimera Mutant } & & \multicolumn{2}{c}{ Human/Mouse Chimera CLDN-5 Mutant } \\
\cline { 3 - 3 } \cline { 6 - 7 } & & $1-5$ & $5-1$ & & D68E & T75A & S151T \\
\hline M11 & Mouse IgG2b & - & +++ & & - & +++ & +++ \\
M48 & Mouse IgG3 & +++ & - & & +++ & +++ & - \\
R2 & Rat IgG2a & - & +++ & & - & +++ & +++ \\
R9 & Rat IgG2b & +++ & - & & +++ & +++ & + \\
\hline
\end{tabular}

,++++ and - indicate strong, weak, and negligible binding reactivity of anti-CLDN-5 antibody against each chimera CLDN.

(Morita et al., 1999; Nitta et al., 2003). This suggests that treatment with CLDN-5 binders may induce severe side effects. However, the expression level of CLDN-5 in peripheral organs and peripheral microvascular endothelial cells is lower than that in brain microvasculature cells (Morita et al., 1999; Rahner et al., 2001). In addition, in the peripheral organs, other CLDNs are also likely involved in the maintenance of TJ integrity and organ homeostasis (Tamura and Tsukita, 2014), and the TJs between peripheral microvascular endothelial cells are not well developed compared with those in the BBB (Morita et al., 1999). This may explain why knockdown of $C l d n-5$ in mice increases the permeation of solutes to the brain without any apparent adverse effects (Campbell et al., 2008; Keaney et al., 2015). Indeed, in brain-injured mice, the knockdown of CLDN-5 mRNA expression by using short interfering RNA leads to improved cognitive outcomes without significant histologic abnormalities in the major organs (Campbell et al., 2012). Consistent with this, we found in the present study that treatment with anti-CLDN-5 mAbs did not result in cytotoxicity in vitro. Taken together, these findings suggest that CLDN-5 is a promising target for the development of drug delivery systems targeting the CNS.

CLDNs form TJ strands via cis-interactions between ECLs in the same cell membrane, and adjacent TJ strands interact via trans-interactions between ECLs of adjacent cells, leading to sealing of the paracellular space (Zihni et al., 2016). The crystal structure of CLDN-15 revealed that the cis-and transinteractions among ECLs may determine the permeability of
A

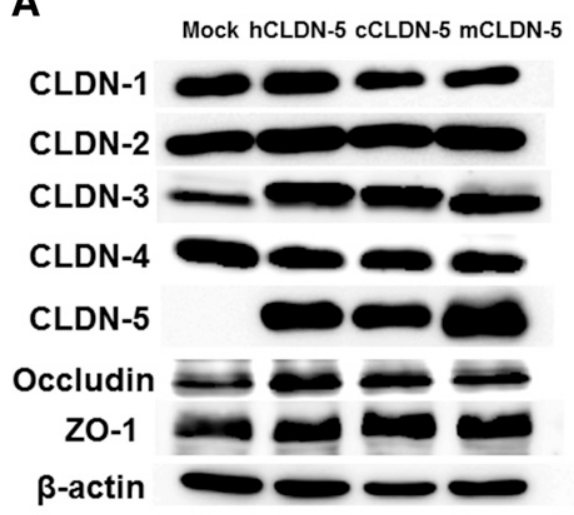

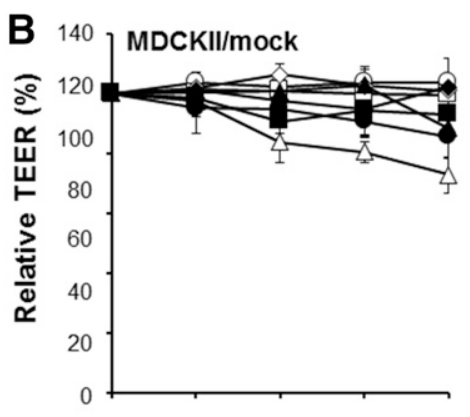
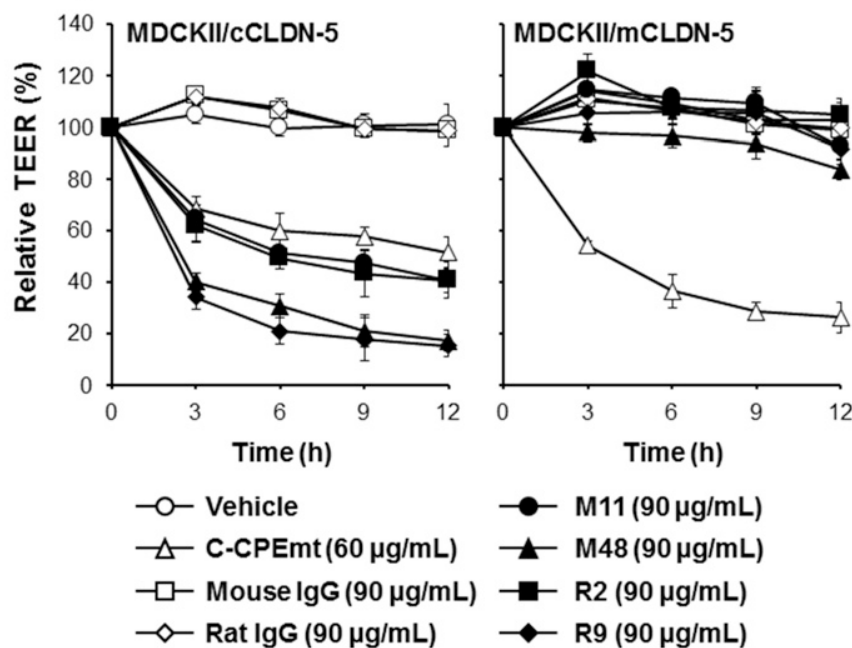

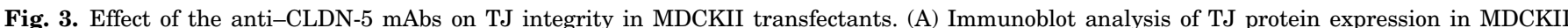

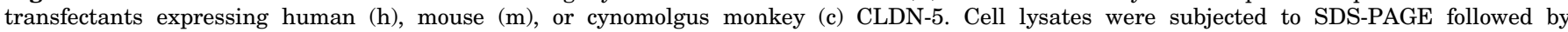

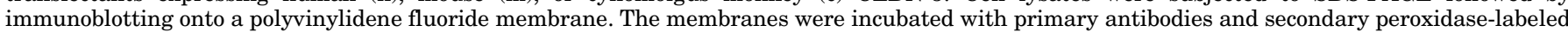

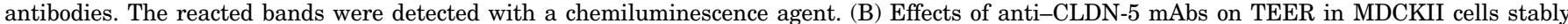

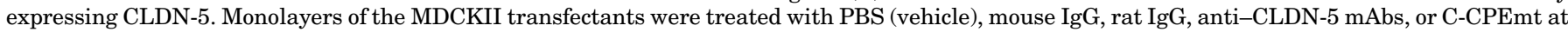

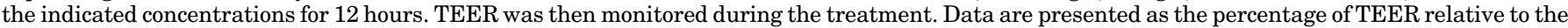
value at 0 hours. Data are representative of three independent experiments. Data are presented as the mean \pm S.D. $(n=3)$. 
A
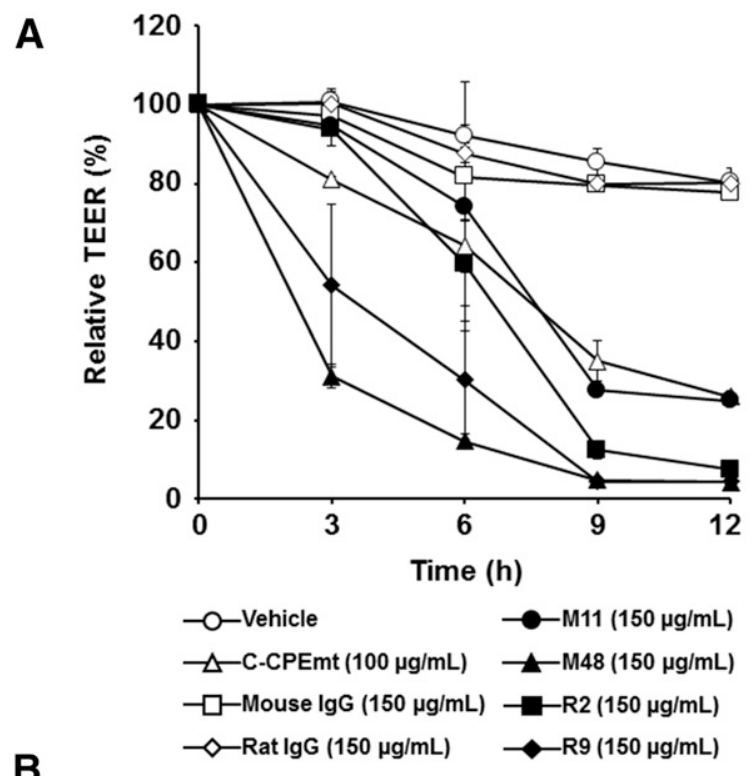

B
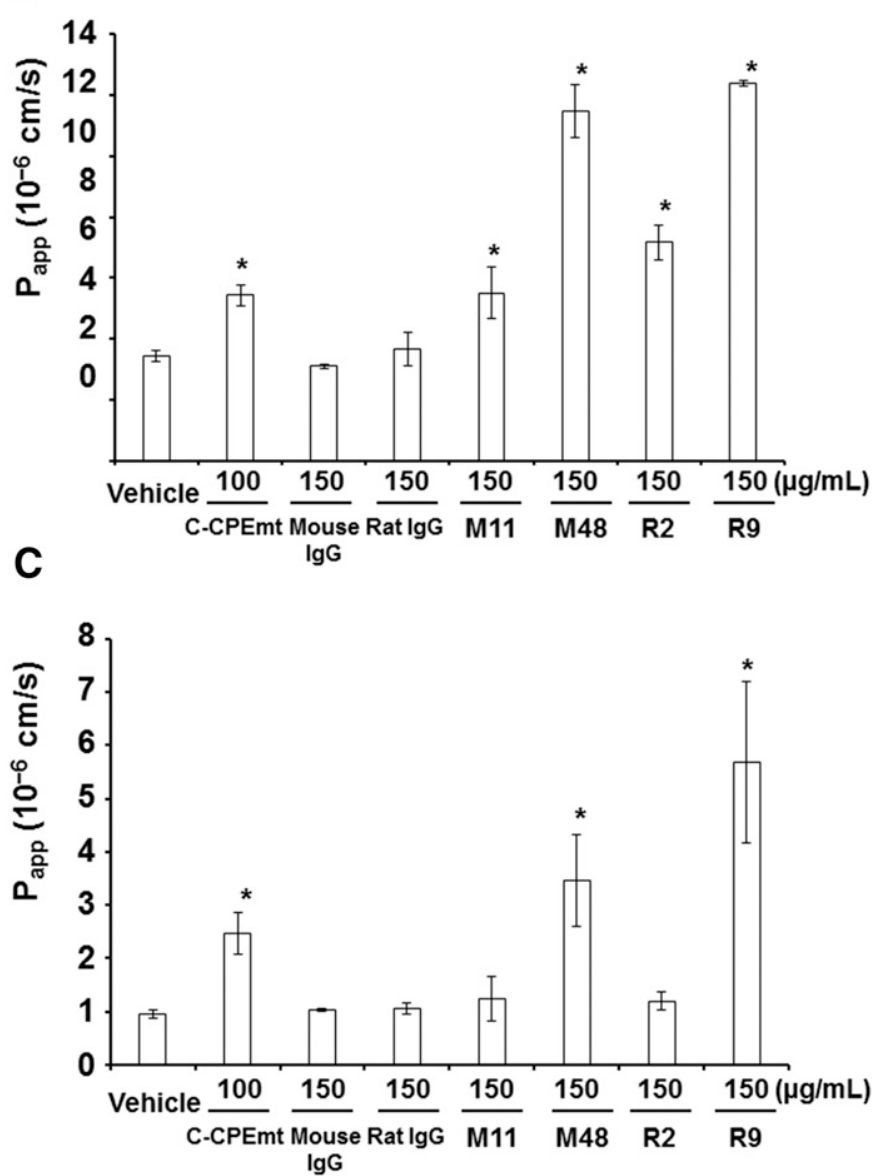

Fig. 4. Effect of the anti-CLDN-5 mAbs on an in vitro model of the BBB. (A) Effects of anti-CLDN-5 mAbs on TEER in a BBB model. Monolayers of cynomolgus monkey brain microvasculature endothelial cells were apically treated with PBS (vehicle), mouse IgG, rat IgG, anti-CLDN-5 mAbs, or C-CPEmt at the indicated concentrations for 12 hours. TEER was monitored during the treatment. Data are expressed as the percentage of TEER relative to the value at 0 hours. Data are representative of three independent experiments. Data are presented as the mean \pm S.D. $(n=3)$. (B and C) Effects of the anti-CLDN-5 mAbs on the permeation of solutes. Cynomolgus monkey brain microvasculature endothelial cells were treated with anti-CLDN-5 mAbs or C-CPEmt at the indicated concentrations for 12 hours. The cells were then incubated on the apical side with a fluorescein dye with a molecular mass of $376 \mathrm{Da}$
TJs (Suzuki et al., 2014, 2015). We previously found that a CLDN-3/-4 binder (C-CPE) enhanced the permeation of solutes across the mucosal membrane, indicating that CLDN binders may enhance permeation via the paracellular pathway by modulating the TJ seal (Kondoh et al., 2005; Uchida et al., 2010). Cysteine 54, cysteine 64, leucine 50, and tryptophan 51 in ECL1 of CLDN-5 are involved in paracellular barrier formation (Wen et al., 2004). Phenylalanine 147, tyrosine 148, glutamine 156, tyrosine 158, and glutamic acid 159 in ECL2 of CLDN-5 are involved in the trans-interactions between cells (Piontek et al., 2008). In the present study, we found that C-CPEmt, M11, M48, R2, and R9 decreased TJ integrity in a model of the BBB. C-CPEmt mainly binds to CLDN-5 at aspartic acid 149/threonine 151 (Protze et al., 2015), M48 and R9 interacted with ECL2 of CLDN-5, and M11 and R2 interacted with ECL1 of CLDN-5. These findings indicate that binders targeting ECL1 and ECL2 of CLDN-5 may prevent the cis- and trans-interactions of CLDN-5 in the TJ seal, resulting in enhancing the permeation of molecules across the BBB.

Treatment of cynomolgus monkey brain endothelial cells with anti-CLDN-5 mAbs altered the localization of CLDN-5 from the borders between cells to the cytosol (Supplemental Fig. 5). This finding is consistent with previous reports that a CLDN-4 binder decreased the amount of CLDN-4 in TJs and a CLDN-1 and CLDN-5 binder increased the intracellular localization of CLDN-1 and CLDN-5 (Staat et al., 2015). Together, these findings suggest that the binding of mAbs to the ECLs of CLDN-5 may induce intracellular uptake of the binder-bound CLDN-5, thereby disrupting the CLDN-5 transinteractions in the TJ seal between adjacent cells and loosening the seal in the paracellular space.

The currently available drug delivery systems targeting the CNS, injection of mannitol and treatment with pulsed sound, disrupt the TJ seal and open the paracellular space to 200 and $50 \mathrm{~nm}$, respectively, leading to the diffusion of substances, such as albumin, into the brain (Rapoport, 2000; Chen and Konofagou, 2014; Kovacs et al., 2017). CLDNs in TJs form charge-selective pores with a diameter of $4 \mathrm{~nm}$ and, therefore, have unique roles in the size- and charge-selective paracellular transport of solutes across the membrane (Furuse and Tsukita, 2006; Van Itallie and Anderson, 2013). In addition, the CLDN-based strands within TJs are dynamic structures that undergo continuous local annealing and breaking (Sasaki et al., 2003). Therefore, the pore-forming and dynamic movements of the TJ seal are determined by the specific combination of CLDNs in the strands, which differs depending on the tissue (Gunzel and Fromm, 2012; Van Itallie and Anderson, 2013). C-CPEmt, a broadly specific CLDN binder to CLDN-1 to CLDN-7, decreased the integrity of the TJ seal in BBB models and intestinal models. In contrast, anti-CLDN-5 mAbs reduced integrity of TJ seal in only BBB models. These findings strongly indicate that the inhibition of CLDNs modulates or controls the paracellular transport of

(B) or with fluorescence-conjugated dextran with an average molecular mass of $4 \mathrm{kDa}(\mathrm{C})$, and the $\mathrm{P}_{\text {app }}$ values from the apical side to the basal side was measured. Data are representative of three independent experiments. Data are presented as the mean \pm S.D. $(n=3)$. $* P<0.05$ vs. vehicle treatment. 


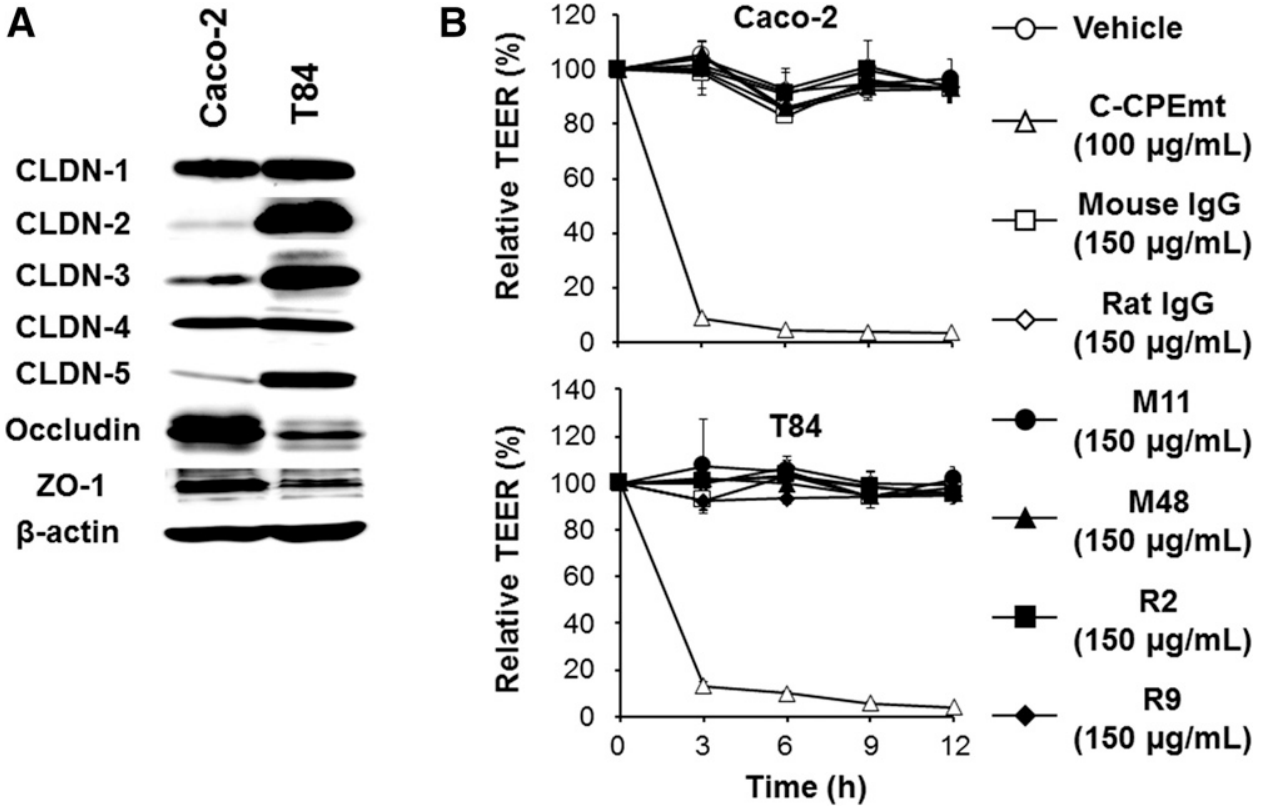

Fig. 5. Effect of anti-CLDN-5 mAbs on TJ integrity in human intestinal cell monolayers. (A) Immunoblot analysis of $\mathrm{TJ}$ proteins in Caco-2 and T84 cells. Cell lysates were subjected to SDS-PAGE followed by immunoblot onto polyvinylidene fluoride membrane. The membranes were incubated with primary antibodies and secondary peroxidase-labeled antibodies. The reacted bands were detected with a chemiluminescence agent. (B) Effects of the anti-CLDN-5 mAbs on TEER in Caco-2 and T84 monolayers. Monolayers of Caco-2 cells and T84 cells were treated with PBS (vehicle), anti-CLDN-5 mAbs, mouse IgG, rat IgG, M11, or C-CPEmt at the indicated concentrations for 12 hours. TEER was monitored during the treatment. Data are expressed as the percentage of TEER relative to the value at 0 hours. Data are representative of three independent experiments. Data are presented as the mean \pm S.D. $(n=3)$. drugs in a size- and charge-dependent manner. Mice in which the expression of CLDN-5 is silenced also allow increased passage of molecules with molecular weights up to $562 \mathrm{Da}$ across the BBB while retaining normal TJ morphology in the BBB (Campbell et al., 2008). The removal of CLDN-5 was left to TJs containing CLDN-12 (Nitta et al., 2003). It is likely that CLDN-5 binders will provide new options for the development of novel drug delivery systems targeting the CNS that are based on modulation, rather than disruption, of the TJ seal.

In conclusion, the anti-CLDN-5 mAbs developed here are the first CLDN-5-specific binders. The present results suggest that CLDN-5 binders are a potential lead for the development of novel drug delivery systems targeting the CNS.

\section{Acknowledgments}

We thank all of the members of our laboratory for their useful comments. We also thank Yuko Fujieda for her excellent technical assistance.

\section{Authorship Contributions}

Participated in research design: Hashimoto, Shirakura, Okada, Takeda, Endo, Sawasaki, and Kondoh.

Conducted experiments: Hashimoto, Shirakura, Takeda, Endo, Tamura, and Sadamura.

Contributed new reagents or analytic tools: Okada, Takeda, and Watari.

Performed data analysis: Hashimoto, Shirakura, Okada, Doi, Yagi, and Kondoh.

Wrote or contributed to the writing of the manuscript: Hashimoto, Okada, Takeda, Sawasaki, Doi, Yagi, and Kondoh.

\section{References}

Akagi T, Shishido T, Murata K, and Hanafusa H (2000) v-Crk activates the phosphoinositide 3-kinase/AKT pathway in transformation. Proc Natl Acad Sci U S A 97:7290-7295

Amasheh S, Schmidt T, Mahn M, Florian P, Mankertz J, Tavalali S, Gitter AH, Schulzke JD, and Fromm M (2005) Contribution of claudin-5 to barrier properties in tight junctions of epithelial cells. Cell Tissue Res 321:89-96.

Betz AL, Firth JA, and Goldstein GW (1980) Polarity of the blood-brain barrier: distribution of enzymes between the luminal and antiluminal membranes of brain capillary endothelial cells. Brain Res 192:17-28.

Campbell M, Hanrahan F, Gobbo OL, Kelly ME, Kiang AS, Humphries MM, Nguyen ATH, Ozaki E, Keaney J, Blau CW, et al. (2012) Targeted suppression of claudin-5 decreases cerebral oedema and improves cognitive outcome following traumatic brain injury. Nat Commun 3:849.
Campbell M, Kiang AS, Kenna PF, Kerskens C, Blau C, O’Dwyer L, Tivnan A, Kelly JA, Brankin B, Farrar GJ, et al. (2008) RNAi-mediated reversible opening of the blood-brain barrier. J Gene Med 10:930-947.

Carpentier A, Canney M, Vignot A, Reina V, Beccaria K, Horodyckid C, Karachi C, Leclercq D, Lafon C, Chapelon JY, et al. (2016) Clinical trial of blood-brain barrier disruption by pulsed ultrasound. Sci Transl Med 8:343re2.

Chen $\mathrm{H}$ and Konofagou EE (2014) The size of blood-brain barrier opening induced by focused ultrasound is dictated by the acoustic pressure. J Cereb Blood Flow Metab 34:1197-1204.

Del Vecchio G, Tscheik C, Tenz K, Helms HC, Winkler L, Blasig R, and Blasig IE (2012) Sodium caprate transiently opens claudin-5-containing barriers at tight junctions of epithelial and endothelial cells. Mol Pharm 9:2523-2533.

Fukasawa M, Nagase S, Shirasago Y, Iida M, Yamashita M, Endo K, Yagi K, Suzuki T, Wakita T, Hanada K, et al. (2015) Monoclonal antibodies against extracellular domains of claudin-1 block hepatitis C virus infection in a mouse model. $J$ Virol $\mathbf{8 9}$ $4866-4879$.

Furuse M (2014) Molecular organization of tricellular tight junctions. Yakugaku Zasshi 134:615-621.

Furuse M and Tsukita S (2006) Claudins in occluding junctions of humans and flies. Trends Cell Biol 16:181-188.

Gribkoff VK and Kaczmarek LK (2017) The need for new approaches in CNS drug discovery: why drugs have failed, and what can be done to improve outcomes. Neuropharmacology 120:11-19.

Günzel D and Fromm M (2012) Claudins and other tight junction proteins. Compr Physiol 2:1819-1852.

Hashimoto Y, Kawahigashi Y, Hata T, Li X, Watari A, Tada M, Ishii-Watabe A, Okada Y, Doi T, Fukasawa M, et al. (2016) Efficacy and safety evaluation of claudin-4-targeted antitumor therapy using a human and mouse cross-reactive monoclonal antibody. Pharmacol Res Perspect 4:e00266.

Heidenreich M and Zhang F (2016) Applications of CRISPR-Cas systems in neuroscience. Nat Rev Neurosci 17:36-44.

Kaitlin KI (2014) CNS drugs have lower regulatory success rate, take longer to develop. Tufts CSDD Impact Reports 16:1-4.

Keaney J, Walsh DM, O'Malley T, Hudson N, Crosbie DE, Loftus T, Sheehan F, McDaid J, Humphries MM, Callanan JJ, et al. (2015) Autoregulated paracellular clearance of amyloid- $\beta$ across the blood-brain barrier. Sci Adv 1:e1500472.

Kondoh M, Masuyama A, Takahashi A, Asano N, Mizuguchi H, Koizumi N, Fujii M, Hayakawa T, Horiguchi Y, and Watanbe Y (2005) A novel strategy for the enhancement of drug absorption using a claudin modulator. Mol Pharmacol 67: $749-756$.

Kovacs ZI, Kim S, Jikaria N, Qureshi F, Milo B, Lewis BK, Bresler M, Burks SR, and Frank JA (2017) Disrupting the blood-brain barrier by focused ultrasound induces sterile inflammation. Proc Natl Acad Sci U S A 114:E75-E84.

Laksitorini M, Prasasty VD, Kiptoo PK, and Siahaan TJ (2014) Pathways and progress in improving drug delivery through the intestinal mucosa and blood-brain barriers. Ther Deliv 5:1143-1163.

Lipinski CA, Lombardo F, Dominy BW, and Feeney PJ (2001) Experimental and computational approaches to estimate solubility and permeability in drug discovery and development settings. Adv Drug Deliv Rev 46:3-26.

Liu Y and Deng W (2016) Reverse engineering human neurodegenerative disease using pluripotent stem cell technology. Brain Res 1638(Pt A):30-41.

Markov AG, Aschenbach JR, and Amasheh S (2015) Claudin clusters as determinants of epithelial barrier function. IUBMB Life 67:29-35.

Morita K, Sasaki H, Furuse M, and Tsukita S (1999) Endothelial claudin: claudin5/TMVCF constitutes tight junction strands in endothelial cells. J Cell Biol 147: 185-194. 
Mosley M, Knight J, Neesse A, Michl P, Iezzi M, Kersemans V, and Cornelissen B (2015) Claudin-4 SPECT imaging allows detection of aplastic lesions in a mouse model of breast cancer. $J$ Nucl Med 56:745-751

Nakagawa S, Deli MA, Kawaguchi H, Shimizudani T, Shimono T, Kittel A, Tanaka $\mathrm{K}$, and Niwa M (2009) A new blood-brain barrier model using primary rat brain endothelial cells, pericytes and astrocytes. Neurochem Int 54:253-263.

Nakajima M, Nagase S, Iida M, Takeda S, Yamashita M, Watari A, Shirasago Y, Fukasawa M, Takeda H, Sawasaki T, et al. (2015) Claudin-1 binder enhances epidermal permeability in a human keratinocyte model. $J$ Pharmacol Exp Ther 354:440-447.

Neesse A, Hahnenkamp A, Griesmann H, Buchholz M, Hahn SA, Maghnouj A, Fendrich V, Ring J, Sipos B, Tuveson DA, et al. (2013) Claudin-4-targeted optical imaging detects pancreatic cancer and its precursor lesions. Gut 62: 1034-1043.

Nitta T, Hata M, Gotoh S, Seo Y, Sasaki H, Hashimoto N, Furuse M, and Tsukita S (2003) Size-selective loosening of the blood-brain barrier in claudin-5-deficient mice. J Cell Biol 161:653-660.

Pardridge WM (2005) The blood-brain barrier: bottleneck in brain drug development. NeuroRx 2:3-14.

Pardridge WM (2007) Drug targeting to the brain. Pharm Res 24:1733-1744.

Pardridge WM and Boado RJ (2012) Reengineering biopharmaceuticals for targeted delivery across the blood-brain barrier. Methods Enzymol 503:269-292.

Piehl C, Piontek J, Cording J, Wolburg H, and Blasig IE (2010) Participation of the second extracellular loop of claudin-5 in paracellular tightening against ions, small and large molecules. Cell Mol Life Sci 67:2131-2140.

Piontek J, Winkler L, Wolburg H, Müller SL, Zuleger N, Piehl C, Wiesner B, Krause G, and Blasig IE (2008) Formation of tight junction: determinants of homophilic interaction between classic claudins. FASEB J 22:146-158.

Protze J, Eichner M, Piontek A, Dinter S, Rossa J, Blecharz KGZ, Vajkoczy P, Piontek J, and Krause G (2015) Directed structural modification of Clostridium perfringens enterotoxin to enhance binding to claudin-5. Cell Mol Life Sci $\mathbf{7 2}$ : 1417-1432.

Rahner C, Mitic LL, and Anderson JM (2001) Heterogeneity in expression and subcellular localization of claudins $2,3,4$, and 5 in the rat liver, pancreas, and gut. Gastroenterology 120:411-422.

Rapoport SI (2000) Osmotic opening of the blood-brain barrier: principles, mechanism, and therapeutic applications. Cell Mol Neurobiol 20:217-230.
Rubin LL and Staddon JM (1999) The cell biology of the blood-brain barrier. Annu Rev Neurosci 22:11-28.

Sasaki H, Matsui C, Furuse K, Mimori-Kiyosue Y, Furuse M, and Tsukita S (2003) Dynamic behavior of paired claudin strands within apposing plasma membranes. Proc Natl Acad Sci U S A 100:3971-3976.

Staat C, Coisne C, Dabrowski S, Stamatovic SM, Andjelkovic AV, Wolburg H, Engelhardt B, and Blasig IE (2015) Mode of action of claudin peptidomimetics in the transient opening of cellular tight junction barriers. Biomaterials 54: $9-20$

Suzuki H, Nishizawa T, Tani K, Yamazaki Y, Tamura A, Ishitani R, Dohmae N, Tsukita S, Nureki O, and Fujiyoshi Y (2014) Crystal structure of a claudin provides insight into the architecture of tight junctions. Science 344:304-307.

Suzuki H, Tani K, Tamura A, Tsukita S, and Fujiyoshi Y (2015) Model for the architecture of claudin-based paracellular ion channels through tight junctions. $J$ Mol Biol 427:291-297.

Takahashi A, Saito Y, Kondoh M, Matsushita K, Krug SM, Suzuki H, Tsujino H, Li X, Aoyama H, Matsuhisa K, et al. (2012) Creation and biochemical analysis of a broad-specific claudin binder. Biomaterials 33:3464-3474.

Tamura A and Tsukita S (2014) Paracellular barrier and channel functions of TJ claudins in organizing biological systems: advances in the field of barriology revealed in knockout mice. Semin Cell Dev Biol 36:177-185.

Uchida H, Kondoh M, Hanada T, Takahashi A, Hamakubo T, and Yagi K (2010) A claudin-4 modulator enhances the mucosal absorption of a biologically active peptide. Biochem Pharmacol 79:1437-1444.

Van Itallie CM and Anderson JM (2013) Claudin interactions in and out of the tight junction. Tissue Barriers 1:e25247.

Wen H, Watry DD, Marcondes MCG, and Fox HS (2004) Selective decrease in paracellular conductance of tight junctions: role of the first extracellular domain of claudin-5. Mol Cell Biol 24:8408-8417.

Zihni C, Mills C, Matter K, and Balda MS (2016) Tight junctions: from simple barriers to multifunctional molecular gates. Nat Rev Mol Cell Biol 17:564-580.

Address correspondence to: Dr. Masuo Kondoh, Graduate School of Pharmaceutical Sciences, Osaka University, Suita, Osaka 565-0871, Japan. E-mail: masuo@phs.osaka-u.ac.jp 